LA W RENCE LIVERMORE NATIONAL LABORATORY
Algorithm Development for the Enhanced Radiological Nuclear Inspection and Evaluation (ERNIE) Tool

T. W. Jacomb-Hood

August 5, 2013 
This document was prepared as an account of work sponsored by an agency of the United States government. Neither the United States government nor Lawrence Livermore National Security, LLC, nor any of their employees makes any warranty, expressed or implied, or assumes any legal liability or responsibility for the accuracy, completeness, or usefulness of any information, apparatus, product, or process disclosed, or represents that its use would not infringe privately owned rights. Reference herein to any specific commercial product, process, or service by trade name, trademark, manufacturer, or otherwise does not necessarily constitute or imply its endorsement, recommendation, or favoring by the United States government or Lawrence Livermore National Security, LLC. The views and opinions of authors expressed herein do not necessarily state or reflect those of the United States government or Lawrence Livermore National Security, LLC, and shall not be used for advertising or product endorsement purposes.

This work performed under the auspices of the U.S. Department of Energy by Lawrence Livermore National Laboratory under Contract DE-AC52-07NA27344. 


\title{
Algorithm Development for the Enhanced Radiological Nuclear Inspection and Evaluation (ERNIE) Tool
}

\author{
Timothy Jacomb-Hood \\ Office of Science, Science Undergraduate Laboratory Internship \\ The Pennsylvania State University \\ Lawrence Livermore National Laboratory \\ Livermore, CA
}

August 9th, 2013

Prepared in partial fulfillment of the requirements of the Office of Science, Department of Energy's Science Undergraduate Laboratory Internship under the direction of S. Labov and K. Nelson in the Global Security Division at Lawrence Livermore National Laboratory. 


\section{TABLE OF CONTENTS}

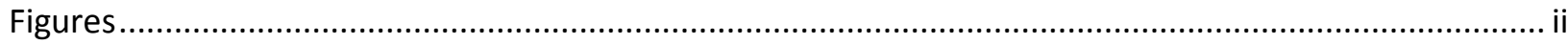

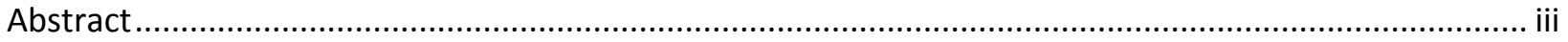

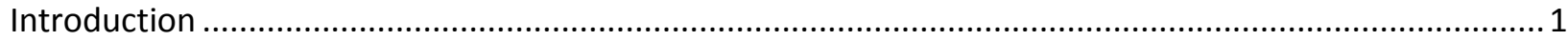

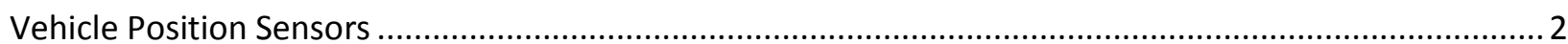

Methodology

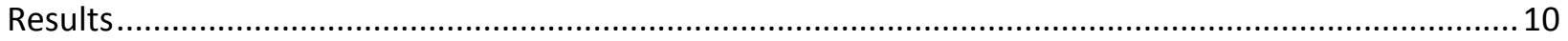

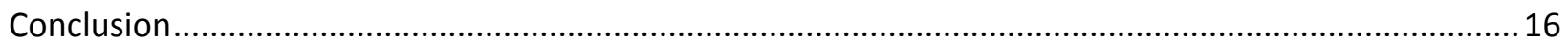

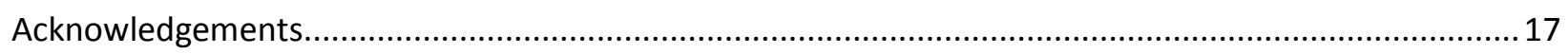




\section{FIGURES}

Figure 1: Truck driving through a RPM, typical sensor beam lines are shown ......................................... 2

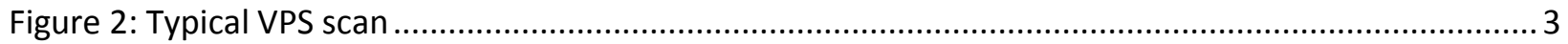

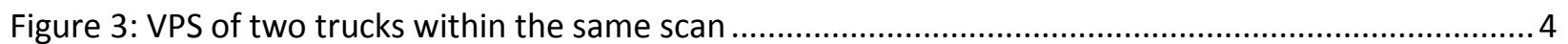

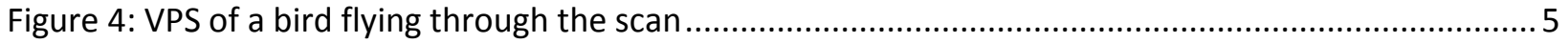

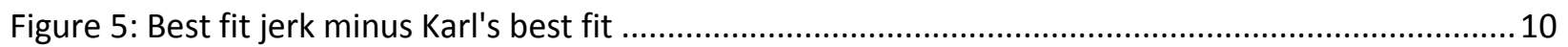

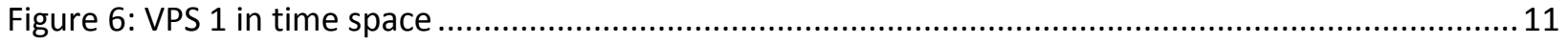

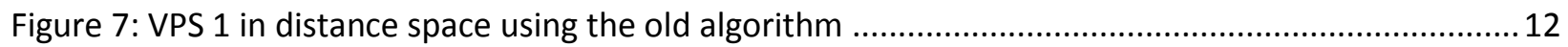

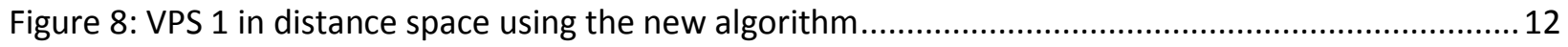

Figure 9: VPS 1 plot showing the correlation between individual VPS scans based on the jerk ...............13

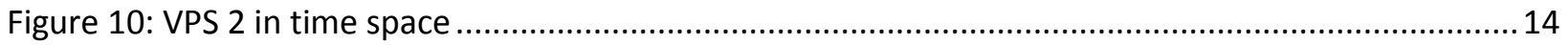

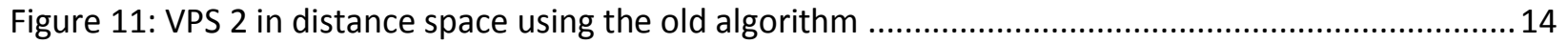

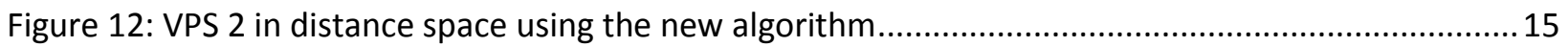

Figure 13: VPS 2 plot showing the correlation between individual VPS scans based on the jerk ............. 15 


\begin{abstract}
At maritime ports in the United States, trucks are driven through Radiation Portal Monitors (RPM) and are measured by four Vehicle Position Sensors (VPS). This project focuses on developing a new and improved algorithm to determine the velocity and motion profile of the trucks.

The VPS data is initially in time space and the new algorithm converts it to distance space assuming that the motion is cubic with respect to time. Applying this new algorithm to the records within the database results in an improvement compared to the original algorithm in $99.0 \%$ of all cases. Only $0.3 \%$ of records showed a noticeable decrease in quality due to the new algorithm.
\end{abstract}

This algorithm, combined with radiation detectors, will be used to locate the radioactive sources within the trucks. This will reduce the time it takes for Customs and Border Patrol Officers to investigate each truck that contains a possible threat. 


\section{INTRODUCTION}

When cargo is shipped to the United States it is screened in a Radiation Portal Monitor (RPM) to determine if dangerous radioactive material is hidden within. The ERNIE project seeks to utilize new algorithms and machine learning to make this process more efficient and be able to better recognize the difference between various types of radioactive sources. ERNIE can distinguish between five different types of radioactive source: Naturally Occurring Radioactive Material (NORM), Industrial, Medical, Nuclear, and Contamination. ERNIE can also tell if there is No Source or if it is an Unknown Source. ERNIE uses gamma detectors to analyze the source and provide a suggestion to the Customs and Border Patrol Officer as to what action should be taken; either investigate the truck further or release the truck.

A part of the RPM is the Vehicle Position Sensors (VPS). The VPS is used to determine physical features of the truck such as the length of the cabin and the length of the trailer. These physical features are combined with the radiation scans to locate the radiation source within the truck. It can also determine if the source is a point source or a distributed source. All of the data collected by the RPM is a function of time and by determining the vehicles motion profile from the VPS data one can convert time to physical location. This allows the Customs and Border Patrol Officers to be much more accurate when they have to physically search a truck looking for the radiation source. 


\section{VEHICLE POSITION SENSORS}

Four vehicle position sensors (VPS) have been built into the radiation monitors (RPM) to be able to determine if the vehicle is within the RPM. Two of the VPS are placed to see the top of the vehicle, and two to see the bottom of the vehicle. When the beam line is broken the system reads that as the truck being within the RPM. The sensors are placed at an angular offset to be sure of seeing the vehicle. Figure 1 below shows how a vehicle would travel through the RPM and the dotted line represent approximate beam paths for each VPS.

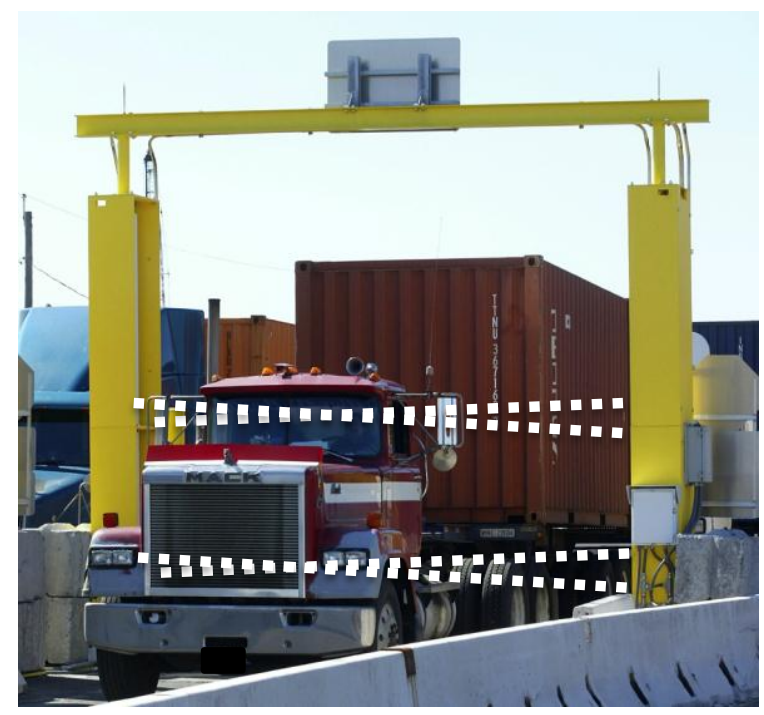

Figure 1: Truck driving through a RPM, typical sensor beam lines are shown

Each sensor generates data showing when that sensor was blocked and when it was unblocked as a function of time. The sensor being blocked correlates to the vehicle being within the RPM, and the sensors being unblocked means that the sensor does not "see" any vehicle.

Figure 2 shows a standard scan of a vehicle as it travels through the RPM. The $x$-axis shows the time relative to when the sensor first starts collecting data and the $y$-axis is used to show a logical 
present/not present within the RPM. The bottom two lines represent the top two sensors, and the top two lines represent the bottom two sensors. The time offset seen between scans is based on the time it takes for the vehicle to travel the distance between the two sensors. This distance is on the order of 1 meter; however, it varies between portal monitors.

This scan is considered a "good" scan because most of the vehicle features are easily determinable by eye. Looking at the Top 1 and Top 2 scans the first area is clearly the truck cabin and the last area is the trailer. The solitary peak between the cabin and trailer is possibly some wire connecting the two. It is a different width in the two different scans which is likely due to the differing angles of the sensor beams. The two sensors will see different faces of the same object causing the peaks to be of different widths. This is an issue that shows up in many different data records but is not as difficult to deal with as the times when one sensor "sees" objects that the other sensor does not. Typically the Bottom 1 and Bottom 2 scans have many more peaks and are more difficult to discern by eye. This is because the area underneath a truck is much more "messy" and there are a lot of objects for the sensors to see.

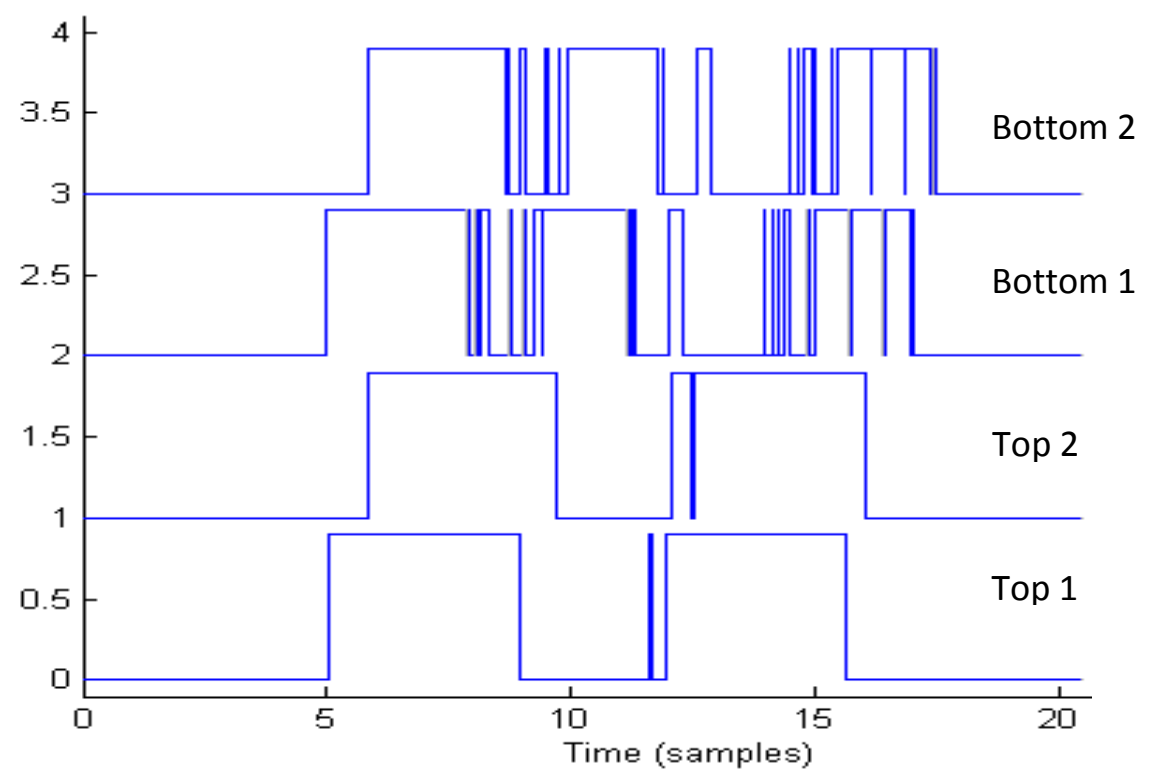

Figure 2: Typical VPS scan 
When looking at the data contained within the database one fact immediately stands out: not all of the data is useable. Some of the records contain data from two trucks driving through the Radiation Portal Monitor (RPM) on the same scan, or a truck that stopped within the RPM. There are also instances of birds flying through the RPM; Figure 3 and Figure 4 show examples of unusable scans.

To be able to deal with the database a program had to be written that would check each individual record and only select useable records. This program was written in concert by Bruno Dueno and Timothy Jacomb-Hood. The selection program used eight criteria to determine whether the record was usable and reduced the initial 7322 records to 6152 usable records.

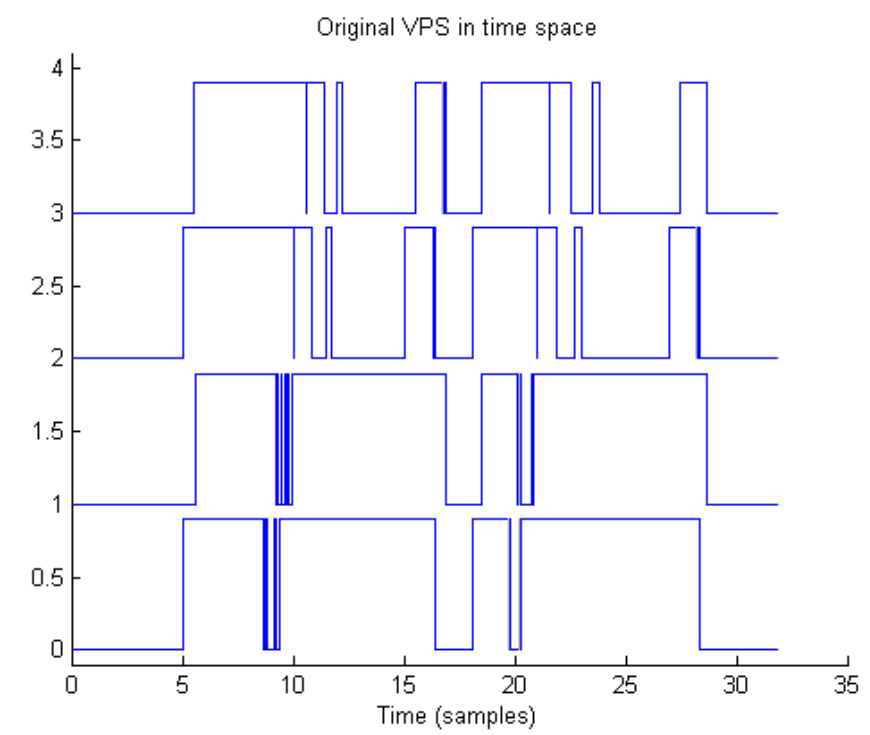

Figure 3: VPS of two trucks within the same scan 


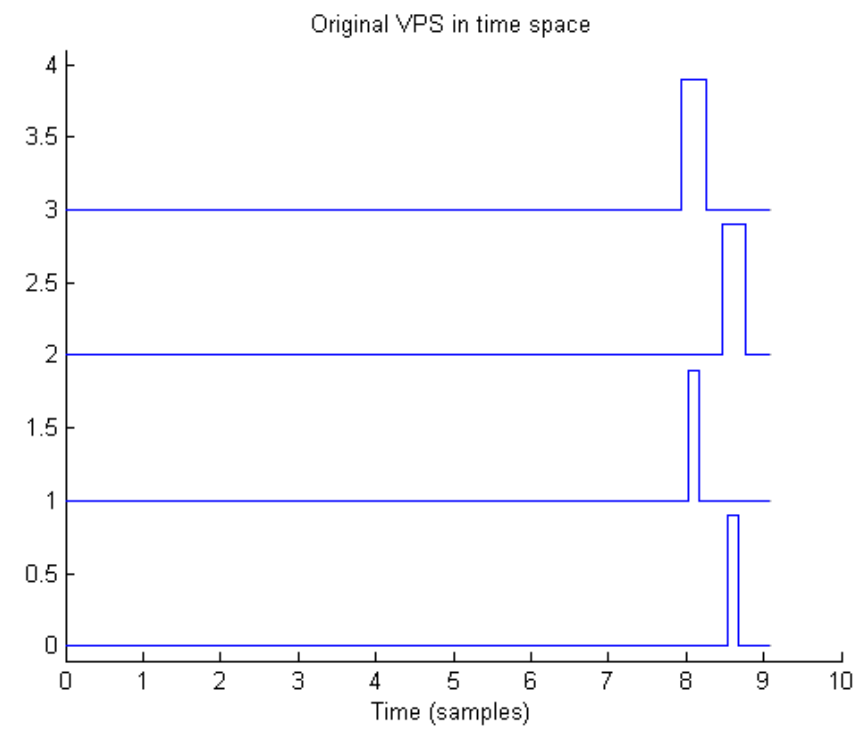

Figure 4: VPS of a bird flying through the scan 


\section{METHODOLOGY}

An algorithm had previously been developed by Dr. Karl Nelson for converting the vehicle position sensor (VPS) data from time space to distance space. His method used a simplified model of the vehicles motion that assumes constant acceleration. This method works very well most of the time although there are instances where it fails at correctly determining the motion profile of the vehicle.

Dr. Nelson wanted to improve the quality of the fit by accounting for non-constant acceleration. Thus the equation describing the vehicles motion now has the jerk term, where jerk is the rate change of acceleration with respect to time.

To solve this new equation of motion it is first assumed that the position of the truck is a continuous function in time and can be modeled by a standard cubic function. The position is then a function of jerk, acceleration, initial velocity, initial position and time.

The position equation could be derived based on either the top sensors or the bottom sensors; however, it is significantly easier to use the top sensors since they generally have fewer features seen and fewer features seen by only one sensor.

To solve the position equation we need four points that are well known in both time and space. Two points will be taken from the Top 1 sensor and two points from the Top 2 sensor. When the vehicle first strikes the Top 1 sensor we define a $t_{1}, x_{1}$, when the vehicle first strikes the Top 2 sensor we define a $t_{2}, x_{2}$, when the truck last leaves the Top 1 sensor we define a $t_{3}, x_{3}$, and when the senor last leaves the Top 2 sensor we define a $t_{4}, x_{4}$.

An initial condition can be set such that $t=0$ is defined as when the truck is first seen by the top sensor and at this time position is defined as zero. Thus $t$ is defined as the length of time past since the vehicle first enters the VPS and $\mathrm{x}$ is defined as the length of the truck measured from the nose of the 
truck. The distance $\mathrm{x}_{2}$ is known because it is the distance traveled between the two sensors, $d$. When the sensors were initially installed the distance between them was recorded.

Mathematically this can be written as:

$$
\begin{gathered}
P(t)=j t^{3}+a t^{2}+v t+x_{0} \\
t_{1}=0, \quad P\left(t_{1}\right)=0 \\
\therefore x_{0}=0 \\
P(t)=j t^{3}+a t^{2}+v_{0} t \\
\\
x_{1}=P\left(t_{1}\right)=0 \\
x_{2}=P\left(t_{2}\right) \\
x_{3}=P\left(t_{3}\right) \\
x_{4}=P\left(t_{4}\right) \\
x_{2}-x_{1}=d \\
P\left(t_{2}\right)-P\left(t_{1}\right)=2 \\
P\left(t_{2}\right)=d
\end{gathered}
$$

The length of the truck, $l$, can be calculated as the distance between either $x_{1}$ and $x_{3}$ or $x_{2}$ and $x_{4}$. Regardless of which sensor is being used the total length of the truck should be the same.

$$
\begin{gathered}
x_{3}-x_{1}=l \\
P\left(t_{3}\right)-P\left(t_{1}\right)=l \\
P\left(t_{3}\right)=l \\
\\
x_{4}-x_{2}=l \\
P\left(t_{4}\right)-P\left(t_{2}\right)=l \\
P\left(t_{4}\right)=P\left(t_{2}\right)+l=d+l
\end{gathered}
$$

In order to solve for the exact position as a function of time three equations are built with four unknowns: j, a, v, I. Initial velocity, acceleration, and truck length can be explicitly solved for and the algorithm will iterate for the jerk term. 


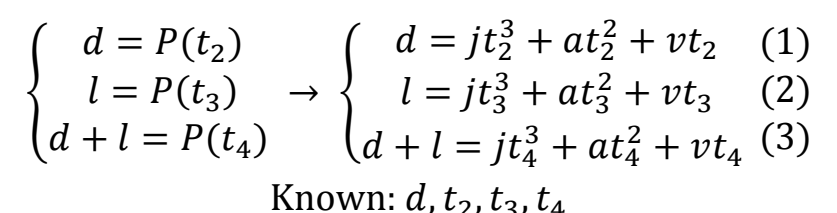

Iterating: $j$

Unknown: $l, v, a$

$$
\begin{gathered}
(1)+(2)=(3) \\
\left(j t_{2}^{3}+a t_{2}^{2}+v t_{2}\right)+\left(j t_{3}^{3}+a t_{3}^{2}+v t_{3}\right)=\left(j t_{4}^{3}+a t_{4}^{2}+v t_{4}\right) \\
j\left(t_{2}^{3}+t_{3}^{3}-t_{4}^{3}\right)+a\left(t_{2}^{2}+t_{3}^{2}-t_{4}^{2}\right)+v\left(t_{2}+t_{3}-t_{4}\right)=0 \cdots(4)
\end{gathered}
$$

Rewrite (1)

$$
a=\frac{x_{2}-j t_{2}^{3}-v t_{2}}{t_{2}^{2}}
$$

(1) into (4)

$$
j\left(t_{2}^{3}+t_{3}^{3}-t_{4}^{3}\right)+\frac{x_{2}-j t_{2}^{3}-v t_{2}}{t_{2}^{2}}\left(t_{2}^{2}+t_{3}^{2}-t_{4}^{2}\right)+v\left(t_{2}+t_{3}-t_{4}\right)=0 \cdots
$$

Rewrite the second term in (5)

$$
\frac{x_{2}-j t_{2}^{3}-v t_{2}}{t_{2}^{2}}\left(t_{2}^{2}+t_{3}^{2}-t_{4}^{2}\right)=\frac{x_{2}-j t_{2}^{3}}{t_{2}^{2}}\left(t_{2}^{2}+t_{3}^{2}-t_{4}^{2}\right)-\frac{v}{t_{2}}\left(t_{2}^{2}+t_{3}^{2}-t_{4}^{2}\right) \cdots
$$

(6) into (5)

$$
\begin{gathered}
j\left(t_{2}^{3}+t_{3}^{3}-t_{4}^{3}\right)+\frac{x_{2}-j t_{2}^{3}}{t_{2}^{2}}\left(t_{2}^{2}+t_{3}^{2}-t_{4}^{2}\right)=v\left[\frac{t_{2}^{2}+t_{3}^{2}-t_{4}^{2}}{t_{2}}-\left(t_{2}+t_{3}-t_{4}\right)\right] \cdots \\
j\left[t_{3}^{2}\left(t_{3}-t_{2}\right)-t_{4}^{2}\left(t_{4}-t_{2}\right)\right]+\frac{x_{2}}{t_{2}^{2}}\left(t_{2}^{2}+t_{3}^{2}-t_{4}^{2}\right)=v\left[\frac{t_{3}^{2}-t_{4}^{2}-t_{2}\left(t_{3}-t_{4}\right)}{t_{2}}\right] \cdots
\end{gathered}
$$

$$
\begin{gathered}
v=v\left(t_{2}, t_{3}, t_{4}, x_{2}, j\right)=\frac{t_{2} j\left[t_{3}^{2}\left(t_{3}-t_{2}\right)-t_{4}^{2}\left(t_{4}-t_{2}\right)\right]+\frac{x_{2}}{t_{2}}\left(t_{2}^{2}+t_{3}^{2}-t_{4}^{2}\right)}{t_{3}^{2}-t_{4}^{2}-t_{2}\left(t_{3}-t_{4}\right)} \\
a=a\left(t_{2}, x_{2}, v, j\right)=\frac{x_{2}-j t_{2}^{3}-v t_{2}}{t_{2}^{2}} \\
l=l\left(t_{3}, v, j, a\right)=j t_{3}^{3}+a t_{3}^{2}+v t_{3}
\end{gathered}
$$


There are now explicit solutions for the initial velocity, acceleration, and truck length as a function of our known times, the distance between the two sensors, and the jerk.

The purpose of this equation is to convert the VPS data from time space to distance space. Most values for jerk will yield a physically impossible position function, thus checking for realism is important. As the trucks move through the RPM their velocity must be always greater than zero. A negative velocity is physically impossible and thus the position functions can be ignored.

If the correct position function is used then the VPS scans will align almost perfectly. The alignment can be measured by the level of overlap seen between the two VPS scans. This alignment is calculated for both the top and bottom VPS and then a weighted average is performed. This "fit" value then ranges from zero to one, with one being a perfectly alignment and zero being no alignment. The fit is measured at every jerk value that provides a realistic motion profile. The best fit is compared to the fit calculated based on the original algorithm to see what level of improvement the new algorithm proves. 


\section{RESULTS}

The records were analyzed using both the new and old algorithms. The best fit using each algorithm was calculated and the difference is plotted in Figure 5. As can be seen, the new algorithm is an improvement over Dr. Nelson's original algorithm in $99.0 \%$ of the 6152 records tested. There are 59 cases when the new algorithm is not an improvement over the original algorithm. However, only 22 cases show a noticeable decrease, reducing the fit by more than 0.001 .

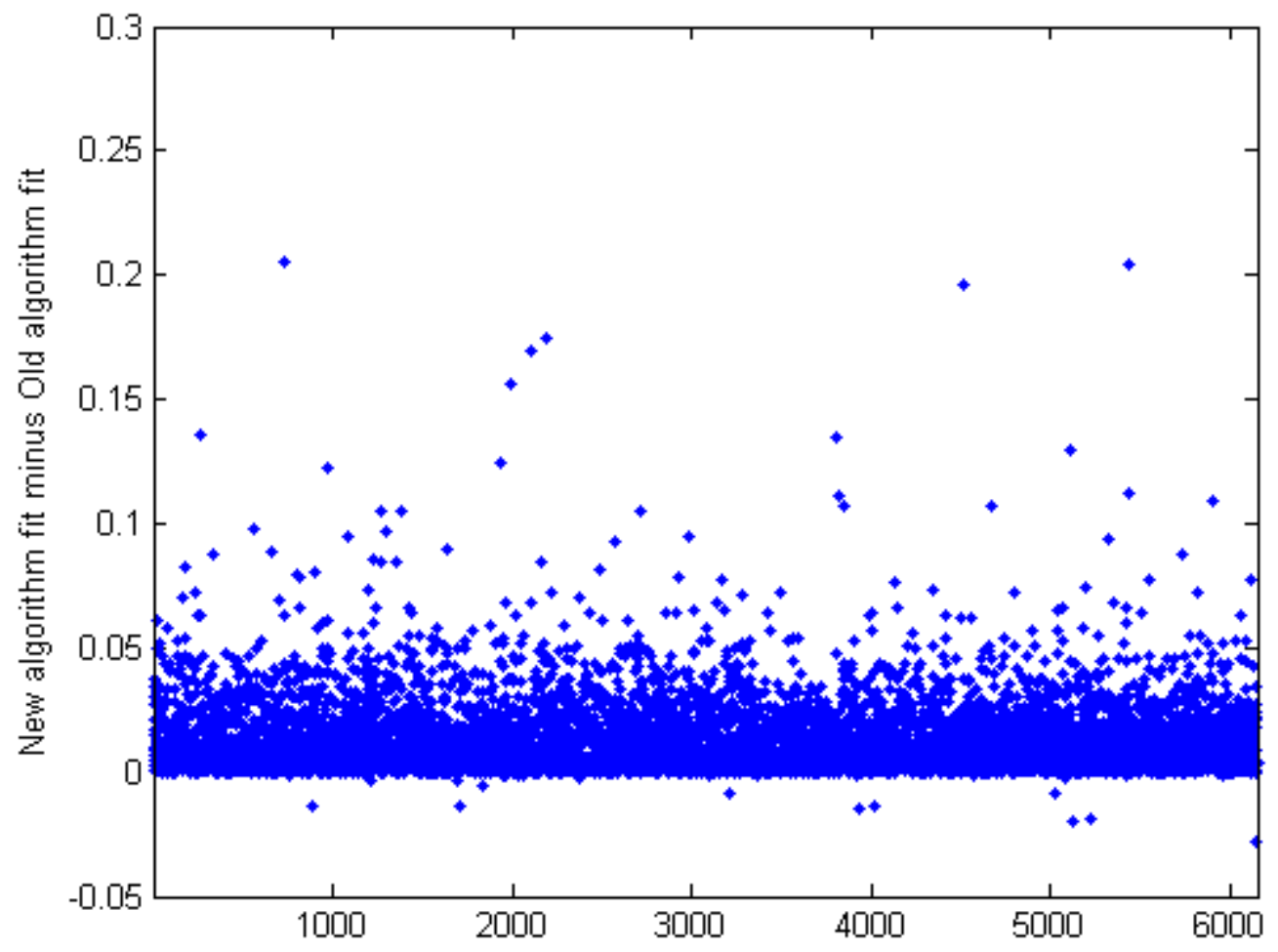

Figure 5: Best fit jerk minus Karl's best fit

Analyzing the cases where the new algorithm is an improvement over the old algorithm shows two cases. One case where there is slight improvement and the second case is where there is significant improvement. 
VPS 1 is a case where the new algorithm shows slight improvement. Figure 6 shows the original scan in time space. This is a clean scan and one can easily see the cabin and trailer. The bottom sensors see a lot more than the top sensors and it is difficult to know the physical meaning of every peak. The original algorithm did a good job in aligning the four VPS profiles but it was not perfect. As can be seen in Figure 7 several of the smaller peaks were not well aligned. The new algorithm generates Figure 8 which does a better job at aligning the peaks and causes the vehicle to become shorter by 1.4 meters. Figure 9 shows the fit as a function of the jerk. There is a very clear peak at $j=2.92 \times 10^{\wedge}-3$ but also a fairly large range of jerks that show improvement over the original algorithm.

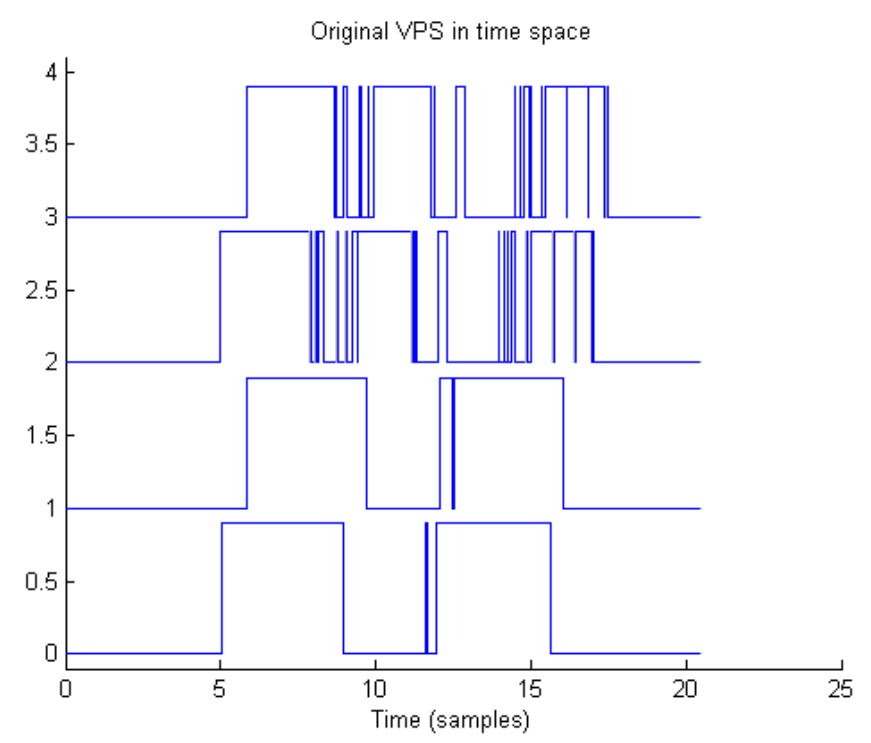

Figure 6: VPS 1 in time space 


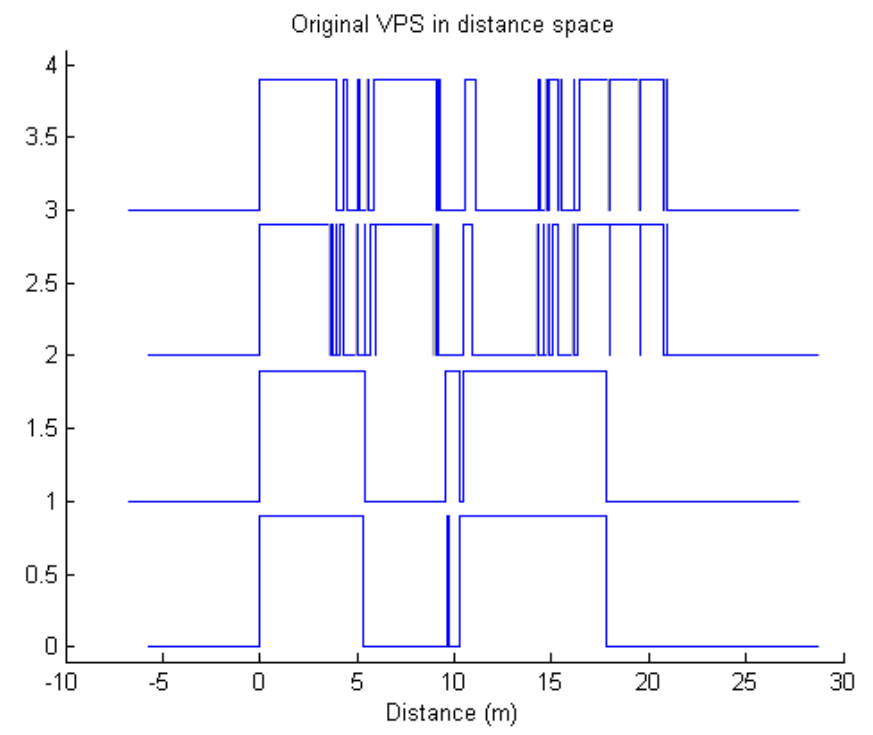

Figure 7: VPS 1 in distance space using the old algorithm

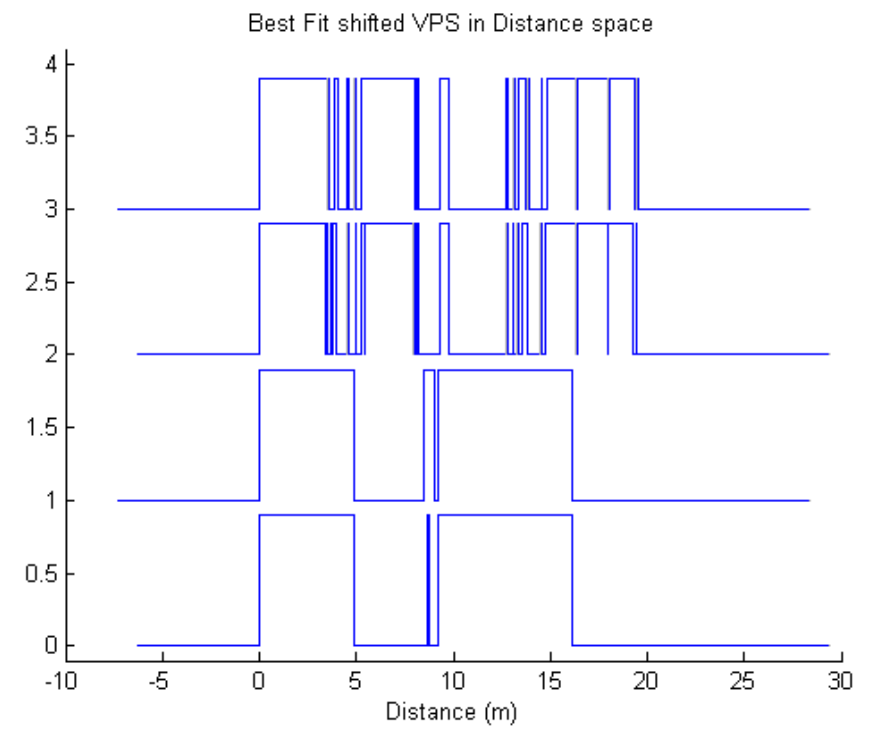

Figure 8: VPS 1 in distance space using the new algorithm 


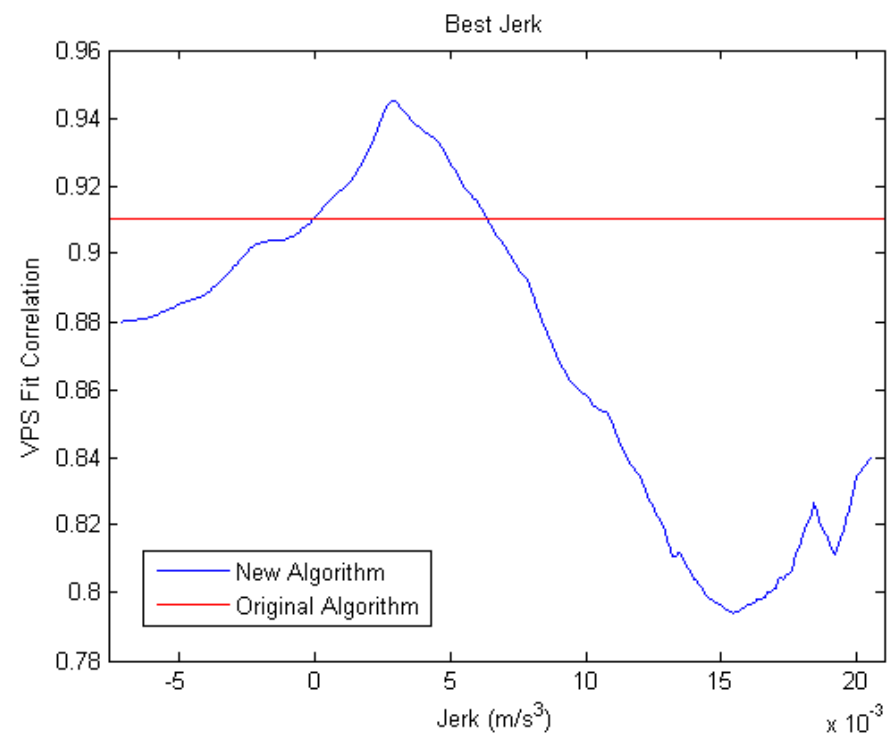

Figure 9: VPS 1 plot showing the correlation between individual VPS scans based on the jerk

VPS 2 is a case where the new algorithm showed significant improvement. In VPS 2 the vehicle did not follow the proper procedure. This truck pulled too far forward in the beginning, sat for an indeterminate amount of time while blocking the first sensor beam, and then started moving and traveled through the rest of the RPM at a normal pace as seen in Figure 10. This makes it appear as if the starting velocity is extremely low. This causes the original algorithm to fail and poorly align the VPS profiles. The original algorithm, Figure 11, aligned the back of the VPS profiles, but failed to correctly align the front end. The new algorithm, Figure 12, manages to align the front end of the VPS even though the front end of the VPS scan is problematic. Figure 13 shows the fit as a function of jerk, for all the jerks that give physically possible motion profiles the new algorithm is a vast improvement over the old algorithm. 


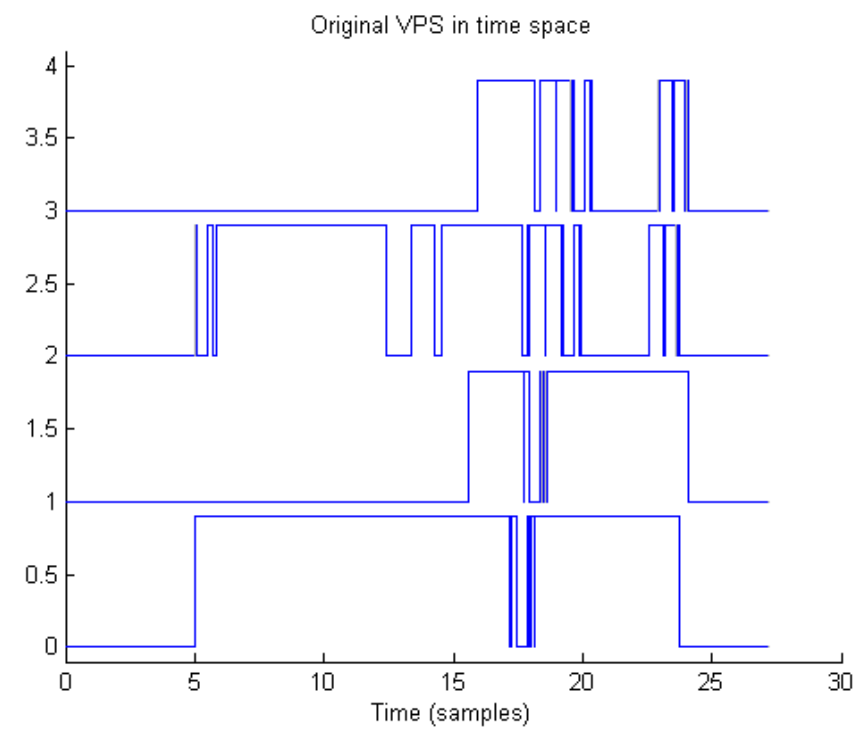

Figure 10: VPS 2 in time space

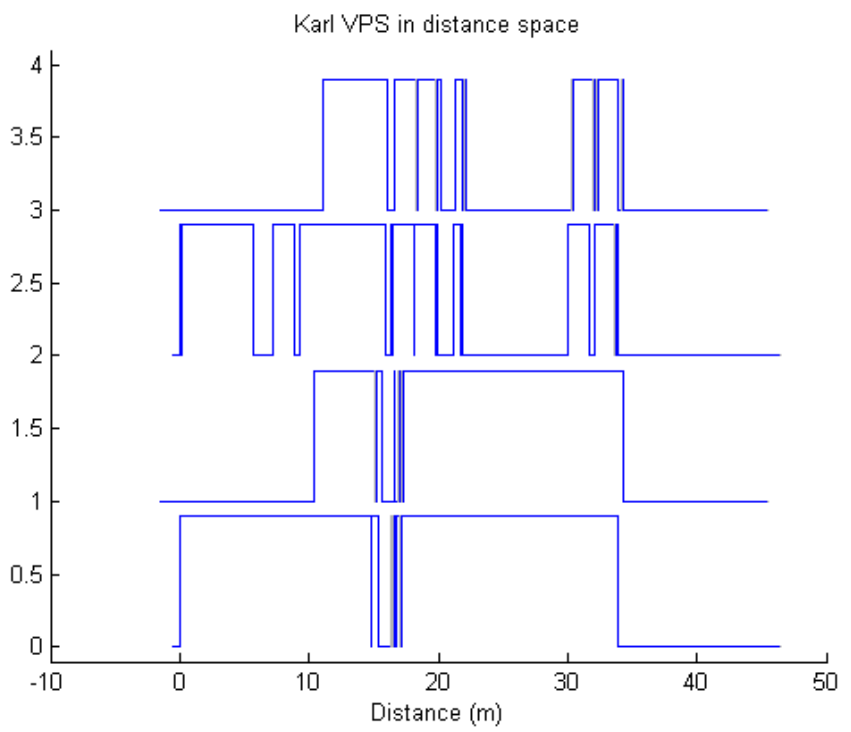

Figure 11: VPS 2 in distance space using the old algorithm 


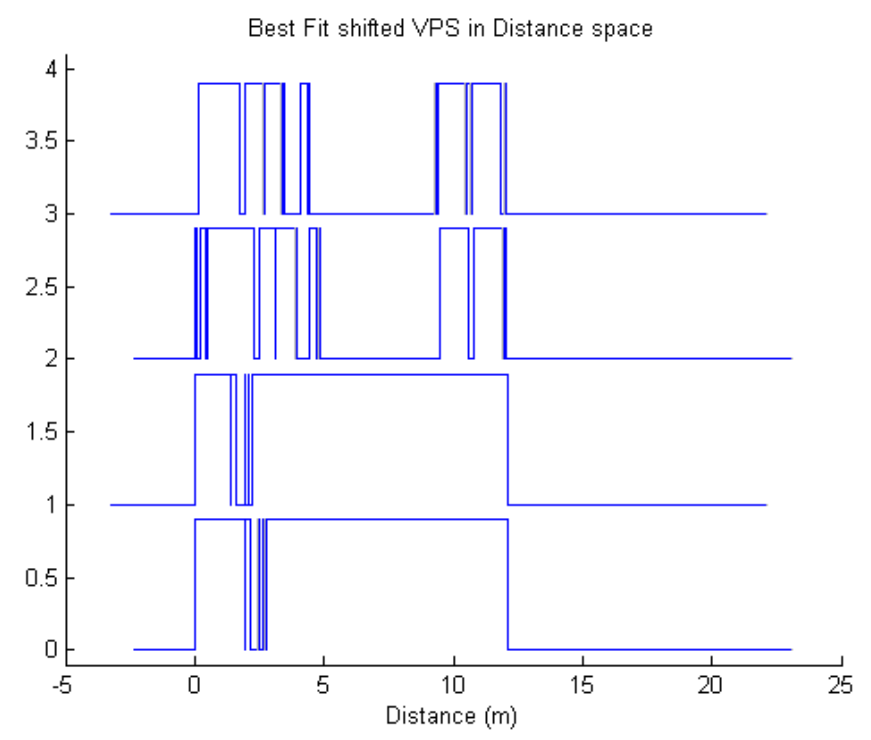

Figure 12: VPS 2 in distance space using the new algorithm

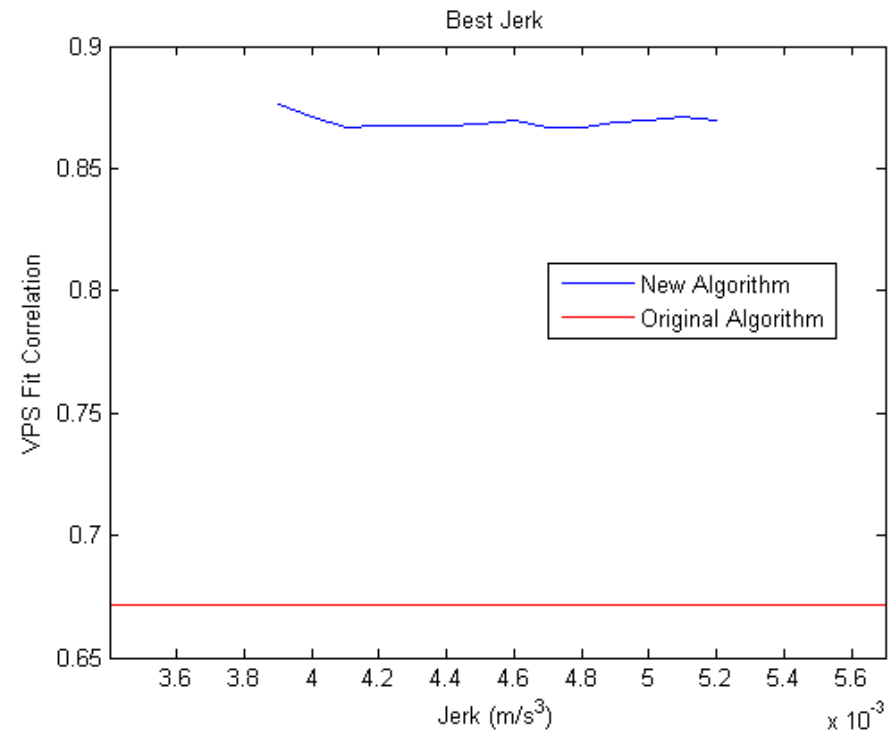

Figure 13: VPS 2 plot showing the correlation between individual VPS scans based on the jerk 


\section{CONCLUSION}

A new algorithm has been developed that is a large improvement over the current algorithm for determining velocity and motion profiles of trucks as they move through Radiation Portal Monitors (RPM). This has improved the fitting of the vehicle velocity profiles in $99 \%$ of records and only noticably decreased the fit in $0.3 \%$ of cases. This improved fit allows the physical features of the trucks to be more accurately determined.

Knowing the physical features with greater accuracy is of great important because the end goal of ERNIE is to accurately be able to determine radiation threats. A threat is determined partly by the gamma intensity measured by the RPM. To accurately know the gamma intensity the gamma background must be known. The background is not directly measured so an average background is used; however, when the trucks go through the RPM the gamma background is suppressed. Larger trucks suppress the background gamma signal more than smaller trucks. This means that, to use an accurate background, the trucks must be catagorized. The improved algorithm allows for better determination of the physical dimensions of the truck which is what the trucks will be categorized on.

Determining background based on truck categorization will further reduce the false positive rate while increasing the detection probability. This will save the Customs and Border Patrol Officers time in checking the false alarms and therefore save them money as they will need less officers on duty. Overall, this will help keep America safe from any external threat trying to enter the nation. 


\section{ACKNOWLEDGEMENTS}

First and foremost I would like to thank Drs. Simon Labov and Karl Nelson for their help and guidance on this project. Without them I would not have been able to make the progresses that I did.

I would also like to thank Bruno Dueno, a student working with me on this project, for his assistance throughout the summer.

Thank you to Jessica Rath our Administrative Assistance for all the help in making our tasks and experience smooth and extraordinary.

Lastly I would like to thank the DOE Office of Science for placing me at Lawrence Livermore National Laboratory this summer. It has been an amazing experience. 